Continuous endurance-type exercise training does not modulate satellite cell content in obese type 2 diabetes patients

Non Peer-reviewed author version

Snijders, Tim; Verdijk, Lex; HANSEN, Dominique; DENDALE, Paul \& van Loon, Luc (2011) Continuous endurance-type exercise training does not modulate satellite cell content in obese type 2 diabetes patients. In: MUSCLE \& NERVE, 43(3), p. 393-401.

DOI: $10.1002 /$ mus.21891

Handle: http://hdl.handle.net/1942/11530 


\title{
CONTINUOUS ENDURANCE-TYPE EXERCISE TRAINING DOES NOT MODULATE SATELLITE CELL CONTENT IN OBESE TYPE 2 DIABETES PATIENTS
}

\author{
TIM SNIJDERS, MSc, ${ }^{1}$ LEX B. VERDIJK, PhD, ${ }^{1}$ DOMINIQUE HANSEN, $\mathrm{PhD}^{2}{ }^{2}$ PAUL DENDALE, MD, PhD, ${ }^{2}$ \\ and LUC J.C. van LOON, PhD' \\ ${ }^{1}$ Department of Human Movement Sciences, NUTRIM School for Nutrition, Toxicology and Metabolism, Maastricht University \\ Medical Centre+, P.O. Box 616, Maastricht 6200 MD, The Netherlands \\ ${ }^{2}$ Faculty of Medicine/Heart Centre Hasselt, Hasselt University, Hasselt, Belgium \\ Accepted 18 August 2010
}

ABSTRACT: Endurance-type exercise training represents a cornerstone in type 2 diabetes treatment. However, the effects of prolonged continuous, endurance-type exercise on muscle fiber characteristics remain equivocal. Fifteen obese male type 2 diabetes patients (61 \pm 6 years) participated in a 6-month continuous, endurance-type exercise program. Muscle biopsies were collected before, and after 2 and 6 months of intervention. Muscle fiber type-specific composition, size, and satellite cell (SC) and myonuclear content were determined by immunohistochemistry. Although continuous endurance-type exercise training lowered total body weight and reduced fat mass, no changes were observed in leg lean mass. At baseline, SC content was significantly lower in type II compared with type I muscle fibers. No change in SC content was observed after exercise training. Continuous endurance-type exercise training lowers fat mass, but it does not increase leg lean mass and/or modulate muscle fiber characteristics in type 2 diabetes patients.

Muscle Nerve 000: 000-000, 2010

Besides diet and medication, exercise is considered to be a cornerstone of effective type 2 diabetes treatment. It has been well established that regular physical exercise represents an effective strategy to prevent and/or treat type 2 diabetes. ${ }^{1}$ In obese type 2 diabetes patients the focus generally lies on the implementation of endurance-type exercise training. ${ }^{2}$ The latter is attributed to the proven efficacy of endurance-type exercise to reduce body fat mass, ${ }^{3-5}$ increase whole-body insulin sensitivity, ${ }^{3,5,6}$ and reduce the risk profile for cardiovascular disease. ${ }^{1}$ Current guidelines from the American Diabetes Association (ADA), European Association for the Study of Diabetes (EASD), and American College of Physicians (ACP) all recognize the therapeutic properties of continuous, endurance-type exercise training. ${ }^{7-9}$ At present, it is recommended that type 2 diabetes patients perform at least $150 \mathrm{~min}$ of moderate-intensity, endurance-type exercise, distributed over at

Abbreviations: ACP, American College of Physicians; ADA, American Diabetes Association; ANOVA, analysis of variance; CSA, cross-sectional area; DAPI, 4',6-diamidino-2-phenylindole; EASD, European Association for the Study of Diabetes; FFM, fat-free mass; FM, fat mass; HR, heart rate; MHC, myosin heavy chain; OGTT, oral glucose tolerance test; SC, satellite cell; $\mathrm{VO}_{2 \max }$, whole-body oxygen uptake; $\mathrm{W}_{\max }$, maximal workload capacity

Key words: immunohistochemistry, insulin sensitivity, muscle stem cells, myonuclei, sarcopenia

Correspondence to: L. J.C. van Loon; e-mail: I.vanloon@hb.unimaas.nl

(C) 2010 Wiley Periodicals, Inc.

Published online in Wiley Online Library (wileyonlinelibrary.com). DOI 10. 1002/mus.21891

Endurance Training and Muscle Fiber Characteristics least 3 days per week, with no more than 2 consecutive days without physical activity. ${ }^{7}$

The loss of skeletal muscle mass and function represents a major factor in the development of insulin resistance and the progression into overt type 2 diabetes. The latter is largely explained by the fact that skeletal muscle tissue is responsible for up to $80 \%$ of whole-body glucose disposal capacity. ${ }^{10}$ As muscle mass largely determines whole-body insulin sensitivity, it is evident that preserving and/ or increasing muscle mass should form one of the main targets in the prevention and/or treatment of type 2 diabetes. Despite the fact that continuous endurance-type exercise training is generally implemented in diabetes intervention programs, there is much debate on the efficacy of continuous endurance-type exercise to stimulate muscle (fiber) hypertrophy in sedentary subjects. Although some studies have reported an increase in muscle fiber size and/ or muscle mass after prolonged endurance-type exercise training in both healthy young and older subjects, ${ }^{11-16}$ others have failed to confirm these findings. ${ }^{17-19}$ Furthermore, whereas some groups have reported changes in skeletal muscle fiber-type distribution after prolonged endurance-type exercise ${ }^{11,13,15,18,20}$ others seem unable to confirm such findings. ${ }^{3,12,14,16,19,21}$ Both animal ${ }^{22}$ and human ${ }^{23}$ studies have indicated that type I muscle fibers are more insulin-sensitive compared with type II muscle fibers. From this perspective, it could be speculated that a reduced percentage of type I muscle fibers contribute to the development of insulin resistance. ${ }^{24}$ Thus, it could also be speculated that an exercise-induced shift toward a higher percentage of type I muscle fibers might support improvements in whole-body insulin sensitivity after prolonged endurance-type exercise training. The impact of prolonged endurance-type exercise on whole-body muscle mass, muscle fiber hypertrophy, and muscle fiber-type distribution remains to be established in sedentary type 2 diabetes patients.

Skeletal muscle hypertrophy after exercise training is accompanied by an increase in the number of muscle stem cells, ${ }^{16,25-31}$ also known as satellite cells (SCs). SCs are located between the 
basal lamina and sarcolemma of myofibers and, upon stimulation, they can proliferate and differentiate into new myonuclei to allow muscle hypertrophy. ${ }^{32,33}$ As such, SCs are instrumental for myofiber growth, repair, and regeneration. ${ }^{34}$ Prolonged resistance type and/or high-intensity intermittent endurance-type exercise training in healthy young ${ }^{25,28,29,35}$ and elderly ${ }^{12,16,27,29-31}$ subjects has been shown to increase SC content in skeletal muscle tissue. So far, the impact of prolonged, continuous endurancetype exercise training on SC content in vivo in type 2 diabetes patients remains largely unexplored. Herein we investigate the impact of prolonged, continuous, endurance-type exercise training on skeletal muscle mass and muscle fiber-type-specific hypertrophy and SC content in long-standing type 2 diabetes patients.

We hypothesized that 6 months of continuous endurance-type exercise would increase muscle mass and induce skeletal muscle fiber hypertrophy with a concomitant muscle fiber-type-specific increase in SCs and myonuclear content in sedentary type 2 diabetes patients. Therefore, body composition and muscle fiber-type-specific hypertrophy, myonuclei, and SC content were assessed prior to and after 2 and 6 months of continuous endurance-type exercise training in obese type 2 diabetes patients.

\section{METHODS}

Subjects. Fifteen male obese type 2 diabetes patients (age $61 \pm 6$ years, body mass index $30.9 \pm$ $0.8 \mathrm{~kg} / \mathrm{m}^{2}$ ) were selected to participate in this study. The medical history of all subjects was evaluated, and an oral glucose tolerance test (OGTT) was performed prior to inclusion. All subjects had been diagnosed with type 2 diabetes for at least 12 months and were using oral blood glucose-lowering medication. Subjects were sedentary and had not participated in any structured exercise training program for at least 5 years. Subjects were informed about the nature and risks of the experimental procedures before written consent was obtained. This study was approved by the local ethics committee of the Virga Jesse Hospital, Hasselt, Belgium.

Study Design. After screening, all eligible subjects participated in a 6-month endurance-type exercise training program. Before, and after 2 and 6 months of continuous endurance-type exercise training, the following parameters were assessed: blood glycated hemoglobin; basal blood glucose, and insulin concentrations; OGTT; whole-body oxidative capacity and maximal workload capacity $\left(\mathrm{VO}_{2 \max }\right.$ test); body composition (dual-energy X-ray absorptiometry scanning); skeletal muscle fiber-type composition; and fiber-type-specific size and SC content. This study is part of a larger pro- ject investigating the clinical benefits of exercise intervention in the treatment of type 2 diabetes.

Diet and Physical Activity Prior to Testing. All subjects maintained habitual physical activity and dietary patterns and refrained from exhaustive physical exercise/labor for at least 3 days prior to each follow-up/test day. On all test days, subjects arrived at the laboratory by car or public transportation after an overnight fast. All subjects recorded dietary intake during 2 days prior to OGTT and repeated the same diet prior to the 2- and 6-month followup measurements. The evening prior to each test day, all subjects received the same standardized meal $(45 \pm 1 \mathrm{~kJ} / \mathrm{kg}$, containing 20 energy $\%$ protein, 47 energy $\%$ fat, and 33 energy $\%$ carbohydrate). Three days prior to the OGTT, patients abstained from taking oral blood glucose-lowering and/or lipid-lowering medication.

Endurance Capacity Assessment. Whole-body oxygen uptake $\left(\mathrm{VO}_{2 \max }\right)$ and maximal workload capacity $\left(\mathrm{W}_{\max }\right)$ were assessed during an exhaustive incremental exercise test on a cycle ergometer (Ergo 1500; Ergofit, Pirmasens, Germany) using a 3-min work-stage protocol. $\mathrm{VO}_{2}$ measurements were performed continuously (CS 200; Schiller, Baar, Switzerland). In addition, cardiac function was monitored with a 12-lead electrocardiogram (ECG), whereas heart rate (HR) was recorded continuously. The measurements were repeated after 2 and 6 months of exercise training.

Exercise Training Intervention Program. All subjects performed three individually supervised continuous endurance-type exercise sessions per week for a 6-month period. During each training session, walking, cycling, and cross-country skiing-type exercise was performed. Each exercise session consisted of 40-min endurance-type exercise at a heart rate that corresponded to an exercise intensity performed at $75 \%$ of the individual baseline $\mathrm{VO}_{2 \max }$. The exercise was standardized by heart rate monitoring (Polar, Oy, Finland). As the target heart rate was maintained throughout the exercise training program and maximal workload capacity increased, energy expenditure during each exercise session gradually increased. After 2 months of exercise training, the relation between heart rate and $\mathrm{VO}_{2 \max }$ was reassessed to recalculate the target heart rate at $75 \%$ $\mathrm{VO}_{2 \max }$. This target was maintained for the remaining 4-month intervention period.

Dual-Energy X-Ray Absorptiometry Scans. Body composition was assessed using dual-energy X-ray absorptiometry (DXA; Lunar DPXL). ${ }^{36}$ Segmental and whole-body fat mass (FM) and fat-free mass (FFM) were determined prior to and after 2 and 6 months of endurance-type exercise training. For 
some subjects, a DXA scan of the entire body could not be performed, as their arms would not fit within the scan area due to the severity of their abdominal obesity. For these subjects $(n=3)$, only regional data were analyzed.

Blood Samples. Subjects arrived at the hospital by car or public transportation and reported to the laboratory at 8:00 A.M. after an overnight fast. After 20 min of supine rest a venous blood sample was collected. Thereafter, a 2-h OGTT was performed. Blood was collected every $30 \mathrm{~min}$ for $2 \mathrm{~h}$. Blood samples were immediately centrifuged at $1000 \times g$ and $4^{\circ} \mathrm{C}$ for $5 \mathrm{~min}$, after which aliquots of plasma were frozen in liquid nitrogen and stored at $-80^{\circ} \mathrm{C}$ until analysis. Blood samples were analyzed for glucose (Beckman Synchron LX 20 Analyzer; Beckman Coulter, Fullerton, California), insulin (Advia Centaur Immunoassay System; Bayer Diagnostics, Tarrytown, New York), and/or glycated hemoglobin $\left(\mathrm{HbA}_{1 \mathrm{c}}\right.$; Hi-Auto A1c Analyzer; Menarini Diagnostics, Florence, Italy).

Muscle Biopsies. Muscle biopsies were collected before, and after 2 and 6 months of training. In the morning, after an overnight fast, subjects arrived at the laboratory by car or public transportation. After local anesthesia, a percutaneous needle biopsy (50$80 \mathrm{mg}$ ) was taken from the middle region of the vastus lateralis muscle, $15 \mathrm{~cm}$ above the patella and approximately $2 \mathrm{~cm}$ away from the fascia. ${ }^{37}$ Muscle biopsies were carefully freed from any visible fat and blood. Thereafter, $\backsim 20 \mathrm{mg}$ of tissue was embedded in Tissue-Tek and rapidly frozen in liquid nitrogencooled isopentane (Sakura Finetek Europe BV, The Netherlands). Muscle biopsies were stored at $-80^{\circ} \mathrm{C}$ for subsequent immunohistochemical analysis to determine myocellular characteristics.

Immunohistochemical Analysis. From all muscle biopsy samples, $5-\mu \mathrm{m}$-thick cross-sections were cut at $-20^{\circ} \mathrm{C}$ using a cryostat. Muscle samples collected before, and after 2 and 6 months of intervention from each individual subject were mounted together on uncoated glass slides, and air-dried for $30 \mathrm{~min}$ at room temperature before being stored at $-20^{\circ} \mathrm{C}$ for subsequent analyses. Muscle cross-sections were stained for muscle fiber distribution, specifically types I, IIa, and IIx. The first antibodies used were directed against laminin (polyclonal rabbit antibody; Sigma Diagnostics, Steinheim, Germany; dilution 1:50), human slow myosin heavy chain (MHC) [A4.840; Developmental Studies Hybridoma Bank (DSHB), Iowa City, Iowa; dilution 1:25], and human fast MHC (IIa) (N2.261, DSHB; dilution 1:25). Staining procedures were as follows. Muscle cross-sections were air-dried for $30 \mathrm{~min}$ at room temperature, then fixed ( $5 \mathrm{~min}$ in acetone).
Again, slides were air-dried and incubated at room temperature with primary antibodies directed against laminin, human slow MHC I and human fast MHC IIa diluted in $0.05 \%$ Tween-phosphatebuffered saline (PBS) for $45 \mathrm{~min}$. Slides were then washed (three times for 5 min in PBS). Thereafter, slides were incubated $30 \mathrm{~min}$ at room temperature with the appropriate secondary antibodies, specifically goat anti-rabbit IgG (Alexa 488), goat antimouse IgM (Alexa 555), and goat anti-mouse IgG1 (Alexa 350; Molecular Probes, Leiden, The Netherlands) diluted in $0.05 \%$ Tween-PBS. After the final washes in PBS, the slides were mounted with mowiol (Calbiochem, Amsterdam, The Netherlands). This staining procedure resulted in laminin being stained green, slow MHC red, and fast MHC blue.

Next, serial cross-sections were stained for muscle fiber SC content. The first antibodies used were directed against laminin (polyclonal rabbit anti-laminin; Sigma) and CD56 (BD Biosciences, San Jose, California). CD56 has been used extensively in various studies for the identification of SC. ${ }^{12,16,25,31,38,39}$ Appropriate secondary antibodies were applied: goat anti-rabbit IgG Alexa Fluor 555 (Molecular Probes; dilution 1:500 and 1:200, respectively) and Avidin-D (Vector Laboratories, Burlingame, California; dilution 1:333), respectively. Nuclei were stained with $0.238 \mu \mathrm{M}$ 4',6-diamidino-2-phenylindole (DAPI; Molecular Probes). Staining procedures were as follows. Muscle crosssections were air-dried for $30 \mathrm{~min}$ at room temperature, then fixed ( $5 \mathrm{~min}$ in acetone). Again, slides were air-dried and incubated at room temperature with primary antibodies directed against laminin (dilution 1:75) and CD56 (dilution 1:40), diluted in $0.05 \%$ Tween-PBS for $45 \mathrm{~min}$. Slides were then washed (three times for 5 min in PBS). Thereafter, the slides were incubated for $30 \mathrm{~min}$ in biotinylated horse anti-mouse IgG (Vector; dilution 1:150) diluted in PBS. After another wash step, slides were incubated for $10 \mathrm{~min}$ with appropriate secondary antibodies, diluted together with DAPI $(0.283 \mu \mathrm{M})$ in $0.05 \%$ Tween-PBS. After a final wash, slides were mounted with cover glasses using mowiol (Calbiochem). Staining procedures resulted in laminin being stained red, nuclei blue, and CD56 green (Fig. 1).

Data Analyses. From the biopsy slides, all images were captured using fluorescence microscopy with a fluorescence microscope (Model E800; Nikon Instruments Europe, Badhoevedorp, The Netherlands) coupled with a progressive scan color CCD camera (Basler A113 C) with a color filter (Bayer). Epifluorescence signal was recorded using a Texas Red excitation filter $(540-580 \mathrm{~nm})$ for either laminin or slow MHC, a DAPI ultraviolet excitation 

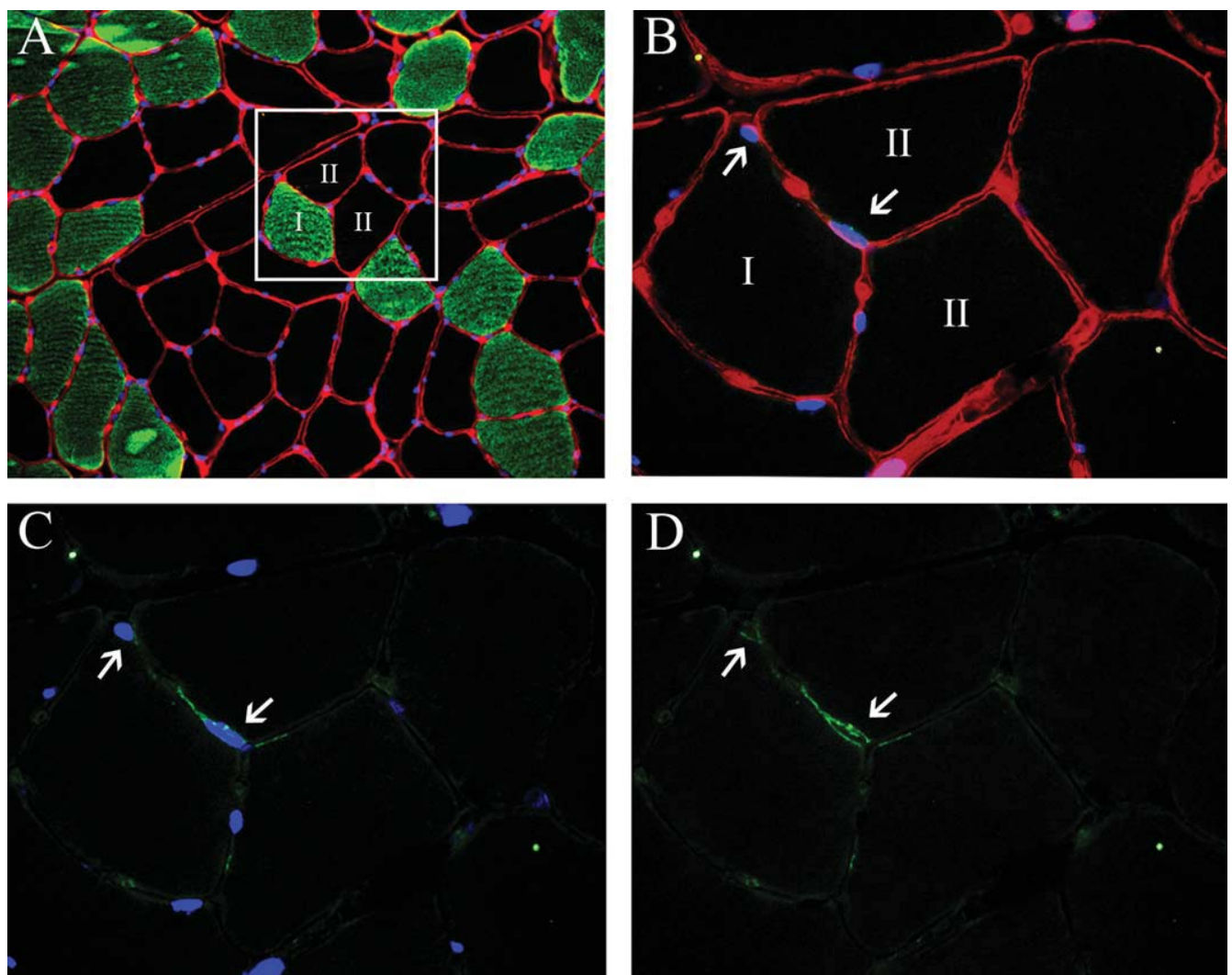

FIGURE 1. Representation of fiber-type-specific analysis of skeletal muscle satellite cells (SC). (A) MHC I+laminin+DAPI staining from serial sections of (B)-(D); the marked area represents the same area as presented in (B)-(D). (B) CD56+laminin+DAPI staining. (C) CD56+DAPI staining. (D) Only CD56 staining. Numbers indicate muscle fiber types I or II. Arrows point at SC.

filter (340-380 nm) for either nuclei or fast MHC, and a fluoroscein isothiocyanate (FITC) excitation filter (465-495 nm) for either CD56 or laminin. Image processing and quantitative analyses were done using Lucia v4.81 software, as described previously. ${ }^{31,39}$ All image recordings and analyses were performed by an investigator blinded to subject coding and/or study design.

From the fiber-type slides, images were captured at $120 \times$ magnification. Laminin was used to determine cell borders and, for all muscle fibers within each image, type I (red), type IIa (blue), and type IIx (black) fibers were identified. Due to the very small number of intermediate-stained muscle fibers $(<2 \%)$, these were excluded from analyses. Within each image the number of muscle fibers and the mean fiber cross-sectional area (CSA) were measured. Fiber circularity was calcu-

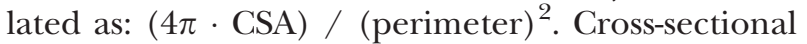
orientation of the fibers was confirmed by fiber circularity. No differences were observed in fiber circularity over time or between fiber types. For muscle fiber size and type distribution, a mean total of $185 \pm 21,172 \pm 17$, and $162 \pm 12$ muscle fibers were analyzed for each muscle biopsy sample collected at baseline, and after 2 or 6 months of endurance-type exercise training, respectively.
From the SC-stained muscle cross-sections, images were captured at a $240 \times$ magnification to allow clear SC localization. Laminin was used to visualize cell borders. SCs were determined at the periphery of each fiber and stained positive for both DNA (DAPI) and CD56. Fiber typing was determined by matching the serial cross-sections stained for MHC I and MHC IIa. Because the number of type IIx muscle fibers was very low, fiber typing was determined by matching only type I and type II muscle fibers in the serial muscle fiber-type slides. As such, the number of SCs per muscle fiber, the proportion of SCs [number of $\mathrm{SC} /$ (number of SC + number of myonuclei) $*$ 100], and the number of SCs per fiber area (in $\mathrm{mm}^{2}$ ) were calculated for the type I and type II muscle fibers separately. In addition, within each image, the number of myonuclei per muscle fiber and the mean fiber area per myonucleus (fiber CSA / number of myonuclei) were assessed for the type I and type II muscle fibers. In line with data presented by Mackey et al., ${ }^{40}$ at least 150 fibers are needed to accurately assess muscle fiber SC content. In this study, we evaluated $283 \pm 18,329 \pm$ 26 , and $310 \pm 26$ muscle fibers for muscle fibertype-specific analyses of SC content for each muscle sample obtained at baseline, and after 2 and 6 
Table 1. Glycemic control and workload capacity.

\begin{tabular}{|c|c|c|c|}
\hline & Baseline & 2 months & 6 months \\
\hline $\mathrm{HbA}_{1 c^{*}}{ }^{*}$ & $7.0 \pm 0.3$ & $6.7 \pm 0.2$ & $6.4 \pm 0.2$ \\
\hline Fasting glucose (mmol/L) & $9.5 \pm 0.6$ & $9.1 \pm 0.6$ & $8.9 \pm 0.5$ \\
\hline Fasting insulin (mU/L) & $19.1 \pm 1.8$ & $17.2 \pm 1.8$ & $16.4 \pm 1.7$ \\
\hline Whole-body $\mathrm{O}_{2}$ uptake capacity $(\mathrm{L} / \mathrm{min})^{*}$ & $2.0 \pm 0.1$ & $2.3 \pm 0.1^{\dagger}$ & $2.3 \pm 0.1^{\dagger}$ \\
\hline Whole-body $\mathrm{O}_{2}$ uptake capacity $(\mathrm{ml} / \mathrm{kg} / \mathrm{min})^{*}$ & $21.7 \pm 1.4$ & $25.5 \pm 1.6^{\dagger}$ & $25.7 \pm 1.5^{\dagger}$ \\
\hline Maximal workload capacity $(\mathrm{W})^{*}$ & $168 \pm 8$ & $183 \pm 8^{\dagger}$ & $186 \pm 9^{\dagger}$ \\
\hline Maximal workload capacity $(\mathrm{W} / \mathrm{kg})^{\star}$ & $1.84 \pm 0.1$ & $2.03 \pm 0.1^{\dagger}$ & $2.08 \pm 0.1^{\dagger}$ \\
\hline
\end{tabular}

Data presented as mean \pm SEM.

${ }^{*}$ Significant time effect $(P<0.05)$.

†Significantly different from baseline value $(P<0.05)$.

months of endurance-type exercise training, respectively.

Statistics. All data are expressed as mean \pm SEM. Kolmogorov-Smirnov tests were applied to confirm that the data were normally distributed. Data were analyzed using a two-way repeated-measures analysis of variance (ANOVA) with time $(0,2$, and 6 months) and fiber type (type I vs. type II, or type I vs. type IIa vs. type IIx) as within-subject factors. In case of a significant interaction, separate analyses were performed for time effects in the type I and type II fibers. Post hoc tests were performed to detect significant differences over time; Bonferroni correction was applied to adjust for multiple testing. Statistical significance was set at $P<0.05$. All calculations were performed using the Statistical Packages for the Social Sciences v15.0 (SPSS, Inc., Chicago, Illinois).

\section{RESULTS}

Subjects. Fifteen male type 2 diabetes patients (age $61 \pm 6$ years, height $1.73 \pm 0.02 \mathrm{~m}$ ) were included. Subjects had been diagnosed with type 2 diabetes for $4.3 \pm 0.7$ years, and all were using oral blood glucose-lowering medication (sulfamides 20\%, biguanides 66\%, glinides 6\%). Body mass was significantly reduced after 2 and 6 months of exercise training (from $92.8 \pm 2.5 \mathrm{~kg}$ to $91.6 \pm 2.5 \mathrm{~kg}$ and $90.9 \pm 2.5 \mathrm{~kg}$, respectively; $P<$ $0.05)$. As a result, body mass index (BMI) decreased significantly $(P<0.05)$ from $30.9 \pm 0.8$ $\mathrm{kg} / \mathrm{m}^{2}$ at baseline to $30.5 \pm 0.8$ and $30.2 \pm 0.8$ $\mathrm{kg} / \mathrm{m}^{2}$ after 2 and 6 months of exercise training, respectively.

Glycemic Control and Performance Capacity. A significant time effect was observed for blood $\mathrm{HbA}_{1 \mathrm{c}}$ levels. Post hoc testing revealed a tendency for a reduction in $\mathrm{HbA}_{1 \mathrm{c}}$ levels from $7.0 \pm 0.3 \%$ to 6.4 $\pm 0.2 \%$ after 6 months of exercise training $(P=$ 0.059 ; Table 1). No significant time effects were observed for fasting plasma glucose and insulin concentrations. Prolonged continuous, endurancetype exercise training significantly increased maxi- mal workload capacity by $12 \pm 4 \%$, from $168 \pm 8$ $\mathrm{W}$ at baseline to $183 \pm 8$ and $186 \pm 9 \mathrm{~W}$ after 2 and 6 months of exercise training, respectively. Furthermore, whole-body oxygen uptake capacity increased from $2.0 \pm 0.1 \mathrm{~L} / \mathrm{min}$ at baseline to 2.3 \pm 0.1 and $2.3 \pm 0.1 \mathrm{~L} / \mathrm{min}$ after 2 and 6 months of continuous, endurance-type exercise training, respectively (Table 1 ).

Body Composition. Changes in body composition, as determined by DXA scan, revealed a significant decrease in body fat percentage from $33.9 \pm 1.5 \%$ at baseline to $32.9 \pm 1.5 \%$ and $31.4 \pm 1.1 \%$ after 2 and 6 months of continuous endurance-type exercise training, respectively $(P<0.05)$. The sum of trunk and leg lean mass increased over the 6-month period from $48.4 \pm 1.0 \mathrm{~kg}$ to $48.8 \pm 1.1 \mathrm{~kg}$ and $49.9 \pm 1.2 \mathrm{~kg}$, respectively $(P<0.05)$. Although no significant changes in trunk lean mass were observed, a significant time effect was observed for leg lean mass $(P=0.024)$. Post hoc testing showed that leg lean mass tended to increase from $18.3 \pm 0.4 \mathrm{~kg}$ at baseline to $19.1 \pm$ $0.6 \mathrm{~kg}$ after 6 months of continuous endurancetype exercise training ( $P=0.054$; Table 2$)$.

Muscle Fiber Type Characteristics. Prior to intervention, the proportion of type I, IIa, and IIx muscle fibers was $51 \pm 3 \%, 42 \pm 3 \%$, and $7 \pm 1 \%$, respectively. The proportion of muscle area occupied by type I, IIa, and IIx fibers was $49 \pm 3 \%, 44$ $\pm 3 \%$, and $7 \pm 2 \%$, respectively. No significant changes were observed over time (Table 3). At baseline, no significant differences were observed between type I and type II muscle fiber CSA, which averaged $5332 \pm 358 \mu \mathrm{m}^{2}$ and $5090 \pm 307 \mu \mathrm{m}^{2}$, respectively. No significant changes in type I or type II muscle fiber CSA were observed during the intervention period (Fig. 2). No differences were observed in myonuclear content or fiber area per myonucleus between type I and II muscle fibers. In addition, no changes were observed in the number of myonuclei per muscle fiber and/or fiber area per myonucleus during the intervention period (Table 4). 
Table 2. Body composition and muscle mass.

\begin{tabular}{lrrr}
\hline & Baseline & 2 months & 6 months \\
\hline${\text { Body fat }(\%)^{\star}}_{\text {Trunk + leg lean mass (kg) }}^{\star}$ & $33.9 \pm 1.5$ & $32.9 \pm 1.5^{\dagger}$ & $31.4 \pm 1.1^{\dagger, \neq}$ \\
Trunk fat (\%) & $48.4 \pm 1.0$ & $48.8 \pm 1.1$ & $49.9 \pm 1.2^{\dagger}$ \\
Trunk fat mass (kg) & $39.9 \pm 1.3$ & $38.9 \pm 1.3$ & $37.1 \pm 1.0^{\dagger, \neq}$ \\
Trunk lean mass (kg) & $20.2 \pm 1.1$ & $19.5 \pm 1.1$ & $18.3 \pm 0.9^{\dagger, \neq}$ \\
Leg fat (\%) & $30.0 \pm 0.7$ & $30.2 \pm 0.7$ & $30.8 \pm 0.7$ \\
Leg fat mass (kg) & $26.3 \pm 1.6$ & $25.3 \pm 1.6^{\dagger}$ & $24.7 \pm 1.5^{\dagger}$ \\
Leg lean mass (kg) & $6.7 \pm 0.6$ & $6.5 \pm 0.6$ & $6.5 \pm 0.6$ \\
\hline
\end{tabular}

Data presented as mean \pm SEM.

*Significant time effect $(P<0.05)$.

${ }^{+}$Significantly different from baseline value $(P<0.05)$.

${ }^{\ddagger}$ Significantly different from 2 -month value $(P<0.05)$.

The number of SCs per type II muscle fiber was substantially lower when compared with the type I muscle fibers $(0.06 \pm 0.01$ vs. $0.10 \pm 0.01$, respectively; $P<0.001$; Table 4 ). Accordingly, the number of SCs per $\mathrm{mm}^{2}$ fiber area, and the percentage of SCs relative to the total number of myonuclei were significantly lower in type II vs. type I muscle fibers at baseline (Table 4). No changes in SC content were observed over time in either type I or type II muscle fibers during the 6-month intervention period. Consequently, SC content remained lower in type II compared with type I muscle fibers after 2 and 6 months of continuous, endurance-type exercise intervention (Table 4).

\section{DISCUSSION}

In this study we have confirmed that 6 months of continuous endurance-type exercise training improves glycemic control, increases whole-body oxidative capacity, and reduces fat mass in obese type 2 diabetes patients. We have extended these findings by the observation that prolonged continuous endurance-type exercise training does not modify skeletal muscle fiber type composition, muscle fiber size, and/or type I or type II muscle fiber myonuclear or SC content in sedentary type 2 diabetes patients. Although no difference between type I and type II muscle fiber size was observed at baseline, we found a lower number of SC in type II compared with type I muscle fibers.

Endurance-type exercise training has been well established as an effective interventional strategy to improve glycemic control, reduce fat mass, and increase whole-body oxidative capacity in obese type 2 diabetes patients. ${ }^{3,4,41}$ Accordingly, we observed a $20 \pm 6 \%$ increase in $\mathrm{VO}_{2 \max }$ after 6 months of endurance-type exercise training in our diabetes patients (Table 1). The latter is in line with previously reported changes after 8-52 weeks of endurance-type exercise training in both healthy subjects and type 2 diabetes patients. ${ }^{3,11-14,16}$ Furthermore, there was a tendency for an increase in leg lean mass $(P=0.054)$ and a decline in blood $\mathrm{HbA}_{1 \mathrm{c}}$ content $(P=0.059)$. In addition, body fat percentage declined significantly from $33.9 \pm$ $1.5 \%$ to $31.4 \% \pm 1.1 \%$ after the exercise program. These findings confirm the efficacy of the applied endurance-type exercise intervention program to improve glycemic control, reduce fat mass, and increase oxidative capacity. Although the reported changes in glycemic control, fat mass, and wholebody oxidative capacity might seem relatively small, they are in line with previously published data in well-controlled type 2 diabetes patients. ${ }^{2,42,43}$

In contrast to the established benefits of prolonged continuous endurance-type exercise training on glycemic control and body composition, there is considerable discrepancy regarding the proposed impact of prolonged continuous endurance-type exercise on skeletal muscle hypertrophy. Whereas some studies have reported significant muscle fiber hypertrophy after 8-52 weeks of endurance-type exercise training in both healthy young and older men and women, ${ }^{11-16}$ others have

\begin{tabular}{|c|c|c|c|c|c|c|}
\hline & \multicolumn{2}{|c|}{ Baseline } & \multicolumn{2}{|c|}{2 months } & \multicolumn{2}{|c|}{6 months } \\
\hline & Fiber \% & CSA\% & Fiber \% & CSA\% & Fiber \% & CSA\% \\
\hline Type I & $51 \pm 2$ & $49 \pm 3$ & $50 \pm 2$ & $50 \pm 2$ & $52 \pm 3$ & $53 \pm 3$ \\
\hline Type Ila & $42 \pm 2$ & $44 \pm 3$ & $44 \pm 1$ & $44 \pm 2$ & $41 \pm 3$ & $40 \pm 3$ \\
\hline Type IIx & $7 \pm 1$ & $7 \pm 2$ & $7 \pm 2$ & $6 \pm 2$ & $8 \pm 3$ & $7 \pm 3$ \\
\hline
\end{tabular}

Data presented as mean \pm SEM; CSA\%, percentage fiber area occupied by type I, Ila, or IIx muscle fibers. No significant differences were observed over time. 


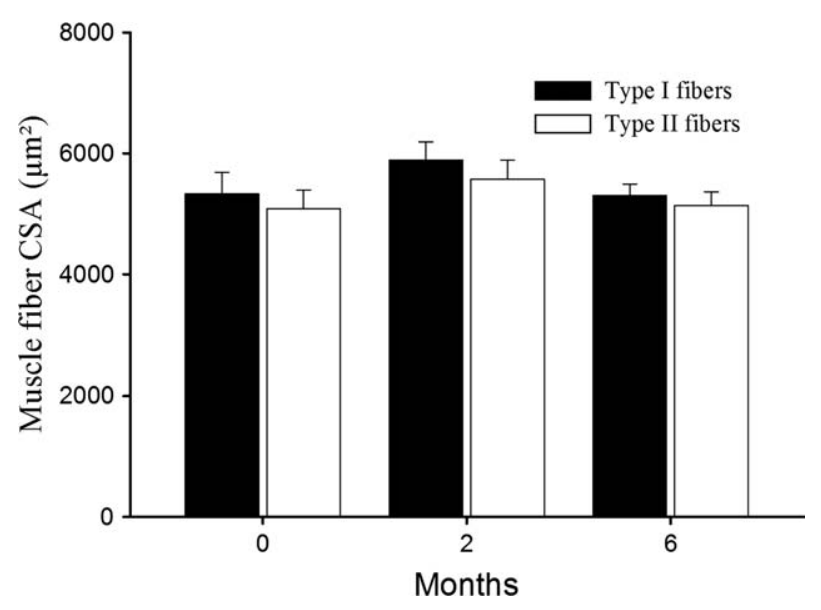

FIGURE 2. Type I and type II muscle fiber cross-sectional area (CSA) prior to and after 2 and 6 months of endurance-type exercise training. No significant differences were observed between type I and type II muscle fiber CSA. No changes in muscle fiber CSA were observed over time.

failed to confirm such changes ${ }^{17-19}$ or even report a concomitant decline in muscle fiber size. ${ }^{20}$ Furthermore, there have been reports of changes in muscle fiber type composition after prolonged endurance-type exercise training. This generally includes a relative shift from type IIx toward more type IIa muscle fibers after endurance-type exercise training in healthy young or older men and women. ${ }^{11,13,15,18,20}$ However, more recent work reported no alterations in fiber type distribution after 14-26 weeks of endurance-type exercise training. ${ }^{3,12,16,19}$ Furthermore, Chomentowski et al. ${ }^{21}$ reported that 4 months of endurance-type exercise training in combination with energy intake restriction does not modify muscle fiber type distribution in type 2 diabetes patients. Our study has shown that prolonged continuous, endurance-type exercise training does not induce muscle fiber hypertrophy and/or modulate muscle fiber type composition in sedentary, obese, type 2 diabetes patients. At baseline, the proportion of type I, IIa, and IIx muscle fibers was $51 \pm 3 \%, 42 \pm 3 \%$, and $7 \pm 1 \%$, respec- tively (Table 3). The latter finding is comparable with those of previous studies in type 2 diabetes patients $^{21,44,45}$ and is in line with observations in healthy young and older men and women. ${ }^{15,16,18,31}$

In this study, we have observed a lower muscle fiber SC content in type II vs. type I muscle fibers (Table 4). However, despite the apparent differences in SC content between muscle fiber types, we did not observe specific type II muscle fiber atrophy in these patients, which is generally observed even in healthy subjects at a more advanced age. ${ }^{16,31,39,46}$ Furthermore, we observed no effect of continuous endurance-type exercise training on muscle fiber size (Fig. 2). A type II muscle fibertype-specific reduction in SC content without a concomitant decline in type II muscle fiber size is of interest, as we have previously shown that type II muscle fiber-type-specific atrophy in senescent muscle is accompanied by a specific decline in type II muscle fiber SC content in elderly ( $76 \pm 1$ years) men. ${ }^{39}$ The observation that type II muscle fiber SC content is already reduced in these subjects at a relatively young age (61 \pm 6 years) might imply that a decline in type II muscle fiber SC content precedes type II muscle fiber atrophy. However, as we did not include a normoglycemic control group it could be speculated that these findings are specific for type 2 diabetes patients only. Apart from muscle fiber size, SC content has been shown to be closely associated with vascular endothelial cells. ${ }^{47,48}$ As muscle fiber capillarization has been hypothesized to govern fiber type-specific SC content, differences in the number of capillaries per fiber type may explain at least some of the observed differences between fiber types. Clearly, further investigations are warranted to investigate the potential functional relationship between muscle fiber SC content, vascular endothelial cells, and muscle fiber size in both health and disease.

For skeletal muscle hypertrophy to occur beyond a certain threshold, the addition of new myonuclei is required. The latter is facilitated by

\begin{tabular}{|c|c|c|c|c|}
\hline & Fiber type & Baseline & 2 months & 6 months \\
\hline \multirow[t]{2}{*}{ Nuclei/fiber } & I & $3.3 \pm 0.2$ & $3.6 \pm 0.3$ & $3.3 \pm 0.3$ \\
\hline & $\|$ & $3.3 \pm 0.3$ & $3.4 \pm 0.3$ & $3.3 \pm 0.3$ \\
\hline \multirow[t]{2}{*}{ Fiber area/nucleus $\left(\mu \mathrm{m}^{2}\right)$} & I & $1651 \pm 111$ & $1813 \pm 192$ & $1724 \pm 181$ \\
\hline & $\|$ & $1606 \pm 126$ & $1773 \pm 183$ & $1703 \pm 201$ \\
\hline \multirow[t]{2}{*}{ SCs/fiber } & I & $0.10 \pm 0.01$ & $0.09 \pm 0.01$ & $0.08 \pm 0.01$ \\
\hline & $\|$ & $0.06 \pm 0.01^{*}$ & $0.07 \pm 0.01^{*}$ & $0.05 \pm 0.01^{*}$ \\
\hline \multirow[t]{2}{*}{$\mathrm{SCs} / \mathrm{mm}^{2}$} & I & $18.2 \pm 1.6$ & $16.3 \pm 2.5$ & $14.6 \pm 1.8$ \\
\hline & ॥ & $11.8 \pm 2.1^{\star}$ & $13.9 \pm 1.8^{\star}$ & $10.1 \pm 1.2^{\star}$ \\
\hline \multirow[t]{2}{*}{ SCs/nuclei (\%) } & I & $2.9 \pm 0.2$ & $2.7 \pm 0.4$ & $2.4 \pm 0.3$ \\
\hline & $\|$ & $1.9 \pm 0.3^{\star}$ & $2.3 \pm 0.3^{\star}$ & $1.5 \pm 0.1^{*}$ \\
\hline
\end{tabular}

Data presented as mean \pm SEM. SCs, satellite cells. No significant changes over time in either type I or II muscle fibers.

${ }^{*}$ Significantly different compared with type I muscle fibers $(P<0.05)$. 
the proliferation of SC and the differentiation of their progeny into new myonuclei that fuse with existing muscle fibers. Muscle fiber hypertrophy has been reported to be accompanied by an increase in SC content after 4-18 weeks of resistance-type exercise training in healthy young and older men and women. ${ }^{16,25-27,29-31}$ In addition, 14 weeks of high-intensity intermittent-type cycling exercise has been shown to increase SC content with a concomitant increase in muscle fiber size in healthy elderly men. ${ }^{12,16}$ We found that 6 months of continuous endurance-type exercise training does not modulate muscle fiber size or myonuclear or SC content. The apparent discrepancy between studies is likely attributed to the different exercise modalities that were applied. So far, studies applying more intense, intermittent-type endurance ${ }^{12,16}$ or resistance-type exercise ${ }^{16,25,27,31}$ have reported substantial increases in muscle fiber size and/or SC content. At present, continuous moderate-intensity endurance-type exercise is generally applied in exercise intervention programs designed for type 2 diabetes patients. However, this study has shown that the latter is not of sufficient impact to stimulate muscle hypertrophy. Nonetheless, we need to be careful when translating these findings beyond the type 2 diabetes population selected in this study. It could be speculated that metabolic disturbances associated with type 2 diabetes interfere with the signaling pathways responsible for $\mathrm{SC}$ activation, proliferation, and renewal. Nonetheless, it is evident that a more intense anabolic stimulus than continuous moderateintensity endurance-type exercise is required to elicit an increase in SC content and augment muscle fiber size in these patients.

In line with these findings, evidence is emerging that suggests the adaptive response generally associated with traditional continuous endurancetype exercise training can be obtained more rapidly through intermittent high-intensity exercise training. ${ }^{49}$ Improvements in whole-body insulin sensitivity, $\mathrm{VO}_{2 \max }$, and mitochondrial biogenesis have been reported to be greater after prolonged intense aerobic interval training when compared with more continuous moderate-intensity exercise training in patients who suffer from metabolic syndrome. ${ }^{50}$ Furthermore, improvements in glycemic control, insulin sensitivity, oxidative capacity, and cardiovascular risk profile after prolonged resistance-type exercise training have been reported to be of a similar magnitude. ${ }^{41,51}$ As both resistance and high-intensity intermittent-type exercise training also augment muscle mass and strength, it seems advisable to include more intense intermittent endurance and/or resistance-type exercise activities in exercise regimens designed to prevent and/or treat type 2 diabetes. ${ }^{2,52}$ The latter is of particular relevance at a more advanced age. The age-related decline in skeletal muscle mass reduces blood glucose disposal capacity, thereby contributing to the development of insulin resistance and type 2 diabetes. ${ }^{53}$ Furthermore, Park et al. ${ }^{54}$ reported that older adults with type 2 diabetes show a more rapid decline in muscle mass and strength. This accelerated decline in muscle mass in elderly type 2 diabetes patients further impairs glucose disposal capacity. ${ }^{54}$ Therefore, more intense intermittent-endurance and/or resistancetype exercise activities should be implemented in exercise intervention programs to attenuate the loss of skeletal muscle mass and increase muscle strength and functional capacity.

We conclude that prolonged continuous, endurance-type exercise training does not augment leg lean mass, induce skeletal muscle fiber hypertrophy, and/or alter muscle fiber type composition in sedentary type 2 diabetes patients. Despite similar type I and II muscle fiber sizes, we observed a significantly lower number of SCs in type II compared with type I muscle fibers at baseline. However, no changes in muscle fiber-type-specific SC content were observed after continuous endurance-type exercise training. As an increase in skeletal muscle mass and strength represents an important target in the prevention and/or treatment of insulin resistance, it is evident that more intense intermittent-endurance and/or resistance-type exercise activities should be implemented in intervention programs designed to prevent and treat type 2 diabetes.

\section{REFERENCES}

1. Snowling NJ, Hopkins WG. Effects of different modes of exercise training on glucose control and risk factors for complications in type 2 diabetic patients: a meta-analysis. Diabetes Care 2006;29:2518-2527.

2. Praet SF, van Loon LJ. Optimizing the therapeutic benefits of exercise in Type 2 diabetes. J Appl Physiol 2007;103:1113-1120.

3. Hansen D, Dendale P, Jonkers RA, Beelen M, Manders RJ, Corluy L, et al. Continuous low- to moderate-intensity exercise training is as effective as moderate- to high-intensity exercise training at lowering blood $\mathrm{HbA}(1 \mathrm{c})$ in obese type 2 diabetes patients. Diabetologia 2009; 52:1789-1797.

4. Lee S, Kuk JL, Davidson LE, Hudson R, Kilpatrick K, Graham TE, et al. Exercise without weight loss is an effective strategy for obesity reduction in obese individuals with and without Type 2 diabetes. $\mathrm{J}$ Appl Physiol 2005;99:1220-1225.

5. Thomas DE, Elliott EJ, Naughton GA. Exercise for type 2 diabetes mellitus. Cochrane Database Syst Rev 2006;3:CD002968.

6. Boule NG, Haddad E, Kenny GP, Wells GA, Sigal RJ. Effects of exercise on glycemic control and body mass in type 2 diabetes mellitus: a meta-analysis of controlled clinical trials. JAMA 2001;286:1218-1227.

7. American Diabetes Association. Standards of medical care in diabetes-2008. Diabetes Care 2008;31 (suppl 1):S12-54.

8. Nathan DM, Buse JB, Davidson MB, Heine RJ, Holman RR, Sherwin $\mathrm{R}$, et al. Management of hyperglycemia in type 2 diabetes: a consensus algorithm for the initiation and adjustment of therapy: a consensus statement from the American Diabetes Association and the European Association for the Study of Diabetes. Diabetes Care 2006; 29:1963-1972

9. Sigal RJ, Kenny GP, Wasserman DH, Castaneda-Sceppa C, White RD. Physical activity/exercise and type 2 diabetes: a consensus statement from the American Diabetes Association. Diabetes Care 2006;29:1433-1438.

10. DeFronzo RA, Ferrannini E, Sato Y, Felig P, Wahren J. Synergistic interaction between exercise and insulin on peripheral glucose uptake. J Clin Invest 1981;68:1468-1474. 
11. Andersen P, Henriksson J. Capillary supply of the quadriceps femoris muscle of man: adaptive response to exercise. J Physiol 1977;270: 677-690.

12. Charifi N, Kadi F, Feasson L, Denis C. Effects of endurance training on satellite cell frequency in skeletal muscle of old men. Muscle Nerve 2003;28:87-92.

13. Coggan AR, Spina RJ, King DS, Rogers MA, Brown M, Nemeth PM, et al. Skeletal muscle adaptations to endurance training in 60- to 70yr-old men and women. J Appl Physiol 1992;72:1780-1786.

14. Gollnick PD, Armstrong RB, Saltin B, Saubert CW, Sembrowich WL, Shepherd RE. Effect of training on enzyme activity and fiber composition of human skeletal muscle. J Appl Physiol 1973;34:107-111.

15. Simoneau JA, Lortie G, Boulay MR, Marcotte M, Thibault MC, Bouchard C. Human skeletal muscle fiber type alteration with high-intensity intermittent training. Eur J Appl Physiol Occup Physiol 1985; 54:250-253.

16. Verney J, Kadi F, Charifi N, Feasson L, Saafi MA, Castells J, et al. Effects of combined lower body endurance and upper body resistance training on the satellite cell pool in elderly subjects. Muscle Nerve 2008;38:1147-1154

17. Hepple RT, Mackinnon SL, Goodman JM, Thomas SG, Plyley MJ. Resistance and aerobic training in older men: effects on VO2peak and the capillary supply to skeletal muscle. J Appl Physiol 1997;82: 1305-1310.

18. Ingjer F. Effects of endurance training on muscle fibre ATP-ase activity, capillary supply and mitochondrial content in man. J Physiol 1979;294:419-432.

19. Putman CT, Xu X, Gillies E, MacLean IM, Bell GJ. Effects of strength, endurance and combined training on myosin heavy chain content and fibre-type distribution in humans. Eur J Appl Physiol 2004;92:376-384

20. Kraemer WJ, Patton JF, Gordon SE, Harman EA, Deschenes MR, Reynolds K, et al. Compatibility of high-intensity strength and endurance training on hormonal and skeletal muscle adaptations. J Appl Physiol 1995;78:976-989.

21. Chomentowski P, Dube JJ, Amati F, Stefanovic-Racic M, Zhu S, Toledo FG, et al. Moderate exercise attenuates the loss of skeletal muscle mass that occurs with intentional caloric restriction-induced weight loss in older, overweight to obese adults. J Gerontol A Biol Sci Med Sci 2009;64:575-580.

22. Henriksen EJ, Bourey RE, Rodnick KJ, Koranyi L, Permutt MA, et al. Glucose transporter protein content and glucose transport capacity in rat skeletal muscles. Am J Physiol 1990;259:E593-598.

23. He J, Watkins S, Kelley DE. Skeletal muscle lipid content and oxidative enzyme activity in relation to muscle fiber type in type 2 diabetes and obesity. Diabetes 2001;50:817-823.

24. Oberbach A, Bossenz Y, Lehmann S, Niebauer J, Adams V, Paschke $\mathrm{R}$, et al. Altered fiber distribution and fiber-specific glycolytic and oxidative enzyme activity in skeletal muscle of patients with type 2 diabetes. Diabetes Care 2006;29:895-900.

25. Kadi F, Schjerling P, Andersen LL, Charifi N, Madsen JL, Christensen LR, et al. The effects of heavy resistance training and detraining on satellite cells in human skeletal muscles. J Physiol 2004;558: $1005-1012$.

26. Kadi F, Thornell LE. Concomitant increases in myonuclear and satellite cell content in female trapezius muscle following strength training. Histochem Cell Biol 2000;113:99-103.

27. Mackey AL, Esmarck B, Kadi F, Koskinen SO, Kongsgaard M, Sylvestersen A, et al. Enhanced satellite cell proliferation with resistance training in elderly men and women. Scand J Med Sci Sports 2007;17: 34-42.

28. Olsen S, Aagaard P, Kadi F, Tufekovic G, Verney J, Olesen JL, et al. Creatine supplementation augments the increase in satellite cell and myonuclei number in human skeletal muscle induced by strength training. J Physiol 2006;573:525-534.

29. Petrella JK, Kim JS, Cross JM, Kosek DJ, Bamman MM. Efficacy of myonuclear addition may explain differential myofiber growth among resistance-trained young and older men and women. Am J Physiol Endocrinol Metab 2006;291:E937-946.

30. Roth SM, Martel GF, Ivey FM, Lemmer JT, Tracy BL, Metter EJ, et al. Skeletal muscle satellite cell characteristics in young and older men and women after heavy resistance strength training. J Gerontol A Biol Sci Med Sci 2001;56:B240-247.

31. Verdijk LB, Gleeson BG, Jonkers RA, Meijer K, Savelberg HH, Dendale $\mathrm{P}$, et al. Skeletal muscle hypertrophy following resistance train- ing is accompanied by a fiber type-specific increase in satellite cell content in elderly men. J Gerontol A Biol Sci Med Sci 2009;64 332-339.

32. Moss FP, Leblond CP. Nature of dividing nuclei in skeletal muscle of growing rats. J Cell Biol 1970;44:459-462.

33. Moss FP, Leblond CP. Satellite cells as the source of nuclei in muscles of growing rats. Anat Rec 1971;170:421-435.

34. Hawke TJ, Garry DJ. Myogenic satellite cells: physiology to molecular biology. J Appl Physiol 2001;91:534-551.

35. Appell HJ, Forsberg S, Hollmann W. Satellite cell activation in human skeletal muscle after training: evidence for muscle fiber neoformation. Int J Sports Med 1988;9:297-299.

36. Haarbo J, Gotfredsen A, Hassager C, Christiansen C. Validation of body composition by dual energy X-ray absorptiometry (DEXA). Clin Physiol 1991;11:331-341.

37. Bergstrom J. Percutaneous needle biopsy of skeletal muscle in physiological and clinical research. Scand J Clin Lab Invest 1975;35: 609-616.

38. Kadi F, Charifi N, Denis C, Lexell J, Andersen JL, Schjerling P, et al. The behaviour of satellite cells in response to exercise: what have we learned from human studies? Pflugers Arch 2005;451:319-327.

39. Verdijk LB, Koopman R, Schaart G, Meijer K, Savelberg HH, van Loon LJ. Satellite cell content is specifically reduced in type II skeletal muscle fibers in the elderly. Am J Physiol Endocrinol Metab 2007;292:E151-157.

40. Mackey AL, Kjaer M, Charifi N, Henriksson J, Bojsen-Moller J, et al. Assessment of satellite cell number and activity status in human skeletal muscle biopsies. Muscle Nerve 2009;40:455-465.

41. Sigal RJ, Kenny GP, Boule NG, Wells GA, Prud'homme D, Fortier M, et al. Effects of aerobic training, resistance training, or both on glycemic control in type 2 diabetes: a randomized trial. Ann Intern Med 2007;147:357-369.

42. Manley S. Haemoglobin Alc-a marker for complications of type 2 diabetes: the experience from the UK Prospective Diabetes Study (UKPDS). Clin Chem Lab Med 2003;41:1182-1190.

43. Praet SF, van Rooij ES, Wijtvliet A, Boonman-de Winter LJ, Enneking $\mathrm{T}$, Kuipers $\mathrm{H}$, et al. Brisk walking compared with an individualised medical fitness programme for patients with type 2 diabetes: a randomised controlled trial. Diabetologia 2008;51:736-746.

44. Cederholm T, Sylven C, Esbjornsson-Liljedahl M, Jansson E. Insulin treatment increases skeletal muscle fibre area in patients with diabetes mellitus type 2. Clin Physiol 2000;20:354-359.

45. Praet SF, Jonkers RA, Schep G, Stehouwer CD, Kuipers H, Keizer HA, et al. Long-standing, insulin-treated type 2 diabetes patients with complications respond well to short-term resistance and interval exercise training. Eur J Endocrinol 2008;158:163-172.

46. Dreyer HC, Blanco CE, Sattler FR, Schroeder ET, Wiswell RA. Satellite cell numbers in young and older men 24 hours after eccentric exercise. Muscle Nerve 2006;33:242-253.

47. Christov C, Chretien F, Abou-Khalil R, Bassez G, Vallet G, Authier FJ, et al. Muscle satellite cells and endothelial cells: close neighbors and privileged partners. Mol Biol Cell 2007;18:1397-1409.

48. Kuang S, Gillespie MA, Rudnicki MA. Niche regulation of muscle satellite cell self-renewal and differentiation. Cell Stem Cell 2008;2: 22-31.

49. Hawley JA, Gibala MJ. Exercise intensity and insulin sensitivity: how low can you go? Diabetologia 2009;52:1709-1713.

50. Tjonna AE, Lee SJ, Rognmo O, Stolen TO, Bye A, Haram PM, et al. Aerobic interval training versus continuous moderate exercise as a treatment for the metabolic syndrome: a pilot study. Circulation 2008;118:346-354.

51. Cauza E, Hanusch-Enserer U, Strasser B, Kostner K, Dunky A, Haber $P$. Strength and endurance training lead to different post exercise glucose profiles in diabetic participants using a continuous subcutaneous glucose monitoring system. Eur J Clin Invest 2005;35:745-751.

52. Praet SF, van Loon LJ. Exercise: the brittle cornerstone of type 2 diabetes treatment. Diabetologia 2008;51:398-401.

53. Pedersen M, Bruunsgaard H, Weis N, Hendel HW, Andreassen BU, Eldrup E, et al. Circulating levels of TNF-alpha and IL-6-relation to truncal fat mass and muscle mass in healthy elderly individuals and in patients with type-2 diabetes. Mech Ageing Dev 2003;124:495-502.

54. Park SW, Goodpaster BH, Strotmeyer ES, Kuller LH, Broudeau R, Kammerer C, et al. Accelerated loss of skeletal muscle strength in older adults with type 2 diabetes: the health, aging, and body composition study. Diabetes Care 2007;30:1507-1512. 